ARTICLE

https://doi.org/10.1038/s41467-019-12101-z

\title{
The mutational landscape of a prion-like domain
}

\author{
Benedetta Bolognesi ${ }^{1,2,6}$, Andre J. Faure (10) ${ }^{1,6}$, Mireia Seuma ${ }^{1,2}$, Jörn M. Schmiedel ${ }^{1}$, \\ Gian Gaetano Tartaglia $1,3,4,5$ \& Ben Lehner (1) 1,3,4
}

Insoluble protein aggregates are the hallmarks of many neurodegenerative diseases. For example, aggregates of TDP-43 occur in nearly all cases of amyotrophic lateral sclerosis (ALS). However, whether aggregates cause cellular toxicity is still not clear, even in simpler cellular systems. We reasoned that deep mutagenesis might be a powerful approach to disentangle the relationship between aggregation and toxicity. We generated $>50,000$ mutations in the prion-like domain (PRD) of TDP-43 and quantified their toxicity in yeast cells. Surprisingly, mutations that increase hydrophobicity and aggregation strongly decrease toxicity. In contrast, toxic variants promote the formation of dynamic liquid-like condensates. Mutations have their strongest effects in a hotspot that genetic interactions reveal to be structured in vivo, illustrating how mutagenesis can probe the in vivo structures of unstructured proteins. Our results show that aggregation of TDP-43 is not harmful but protects cells, most likely by titrating the protein away from a toxic liquid-like phase.

\footnotetext{
${ }^{1}$ Center for Genomic Regulation (CRG), The Barcelona Institute of Science and Technology, Doctor Aiguader 88, 08003 Barcelona, Spain. ${ }^{2}$ Institute of Bioengineering of Catalonia (IBEC), The Barcelona Institute of Science and Technology, Barcelona, Spain. ${ }^{3}$ Universitat Pompeu Fabra (UPF), Barcelona, Spain. ${ }^{4}$ Institució Catalana de Recerca i Estudis Avançats (ICREA), Passeig Lluís Companys 23, 08010 Barcelona, Spain. ${ }^{5}$ Department of Biology 'Charles Darwin', Sapienza University of Rome, P.le A. Moro 5, Rome 00185, Italy. ${ }^{6}$ These authors contributed equally: Benedetta Bolognesi, Andre J. Faure. Correspondence and requests for materials should be addressed to B.B. (email: bbolognesi@ibecbarcelona.eu) or to B.L. (email: ben.lehner@crg.eu)
} 
T he conversion of specific proteins into insoluble aggregates is a hallmark of many neurodegenerative disorders, including Alzheimer's, Parkinson's, Huntington's, and Amyotrophic Lateral Sclerosis (ALS) with dominantly inherited mutations in aggregate-forming proteins causing rare familial forms of these diseases ${ }^{1-6}$. However, both in humans and in animal models, there is often only a weak association between the presence of aggregates and disease progression ${ }^{7-9}$. Indeed, multiple therapeutic approaches that reduce the formation of aggregates have failed at different stages of development ${ }^{10-12}$. On the other hand, there is increasing evidence that alternative protein assemblies generated during or in parallel to the aggregation process may be toxic ${ }^{13-17}$. Despite evidence that cellular damage may be induced either before, after or independent of the formation of insoluble aggregates, the latter are still widely assumed to be pathogenic in many neurodegenerative diseases ${ }^{18,19}$.

For many proteins, aggregation depends critically on intrinsically disordered regions with a low sequence complexity resembling that of infectious yeast prions. These prion-like domains (PRDs) are also enriched in proteins that can form liquid-like cellular condensates ${ }^{20-22}$ through liquid-demixing. This is a concentration-dependent process through which proteins can separate into two coexisting liquid phases and it has been extensively characterized both in vitro and in the cytoplasm ${ }^{23}$. In several proteins PRDs are necessary and sufficient for liquid-liquid demixing 23,24 . At least in vitro, insoluble aggregates can nucleate from more liquid phases ${ }^{24-26}$, leading to the suggestion that liquid de-mixed states can mature into pathological aggregates ${ }^{19}$.

Disordered regions ${ }^{27}$ and low-complexity sequences ${ }^{28}$ are also enriched in dosage-sensitive proteins that are toxic when their concentration is increased. At least for one model protein that has been tested, however, it is the formation of a concentrationdependent liquid-like phase-not aggregation-that causes cellular toxicity ${ }^{28}$. Similarly, the toxicity of two mutant forms of the prion Sup 35 could be explained only on the basis of their ability to populate a non-aggregate, liquid-like state $\mathrm{e}^{20,29}$.

Cytoplasmic aggregates of the TAR DNA-binding protein 43 (TDP-43) are a hallmark of ALS, present in 97\% of post-mortem samples $^{2,30}$. TDP-43 aggregates are also present at autopsy in nearly all cases of frontotemporal dementia (FTD) that lack tau-containing inclusions (about half of all cases of FTD which is the second most common dementia) $)^{31}$. TDP-43 aggregates also represent a hallmark of inclusion body myopathy, and a secondary pathology in Alzheimer's, Parkinson's, and Huntington's disease ${ }^{31-33}$. However, TDP-43 aggregates are also observed-albeit at low frequency-in control samples ${ }^{34}$ and, in vitro, TDP-43 can form both amyloid aggregates and liquid condensates ${ }^{35-39}$. Mutations in TDP-43 cause $\sim 5 \%$ of familial ALS (fALS) cases ${ }^{8,40}$, with these mutations reported to interfere with nuclear-cytoplasmic transport, RNA processing, splicing, and protein translation ${ }^{7,41-46}$. However, despite extensive investigation, the molecular form of the protein that causes cellular toxicity is still unknown ${ }^{7,47}$.

We reasoned that systematic ('deep') mutagenesis could be an unbiased approach to identify and investigate the toxic species of proteins ${ }^{48-50}$. A map of which amino acid (AA) changes increase or decrease the toxicity of a protein to a cell should, if sufficiently comprehensive, clarify both the properties of the protein and its in vivo conformational states associated with toxicity ${ }^{51}$. The effects of a small number of mutations on TDP-43 toxicity or aggregation have been previously reported ${ }^{15,35,52-55}$. However, on the basis of a handful of mutations, the relationship between aggregation and toxicity is far from clear.

Here we show by quantifying the effects of $>50,000$ mutations in the PRD of TDP-43 that increasing hydrophobicity and aggregation strongly reduce the toxicity of this protein in yeast. Moreover, mutations that increase the toxicity of TDP- 43 actually promote the formation of dynamic liquid-like cytoplasmic condensates. Mutations have their strongest effects in a central 'hotspot' region of the PRD TDP-43. The patterns of genetic interactions in double mutants in this region reveal that this 'unstructured' region is actually structured in vivo. Our results illustrate how deep mutagenesis can be used to probe the sequence-function relationships and the in vivo structures of 'disordered' proteins. We propose that aggregation of TDP-43 is not harmful but actually protects cells, most likely by titrating protein from a toxic liquid-like phase.

\section{Results}

Deep mutagenesis of the TDP-43 prion-like domain. We used error-prone oligonucleotide synthesis to comprehensively mutate the PRD of TDP-43. We introduced the library into Saccharomyces cerevisiae cells, induced expression and used deep sequencing before and after induction to quantify the relative effects of each variant on growth in three biological replicates (Fig. 1a). After quality control and filtering (Supplementary Fig. la and c), the dataset quantifies the relative toxicity of 1,266 single and 56,730 double amino acid (AA) changes in the PRD with high reproducibility (Fig. 1b, Supplementary Fig. 1d and e). The toxicity scores also correlate very well with the toxicity of the same variants retested in the absence of competition (Fig. 1c).

The toxicity of both single and double mutants has a tri-modal distribution (Fig. 1d, Supplementary Fig. 2a and c), with 18,023 variants more toxic and 16,152 variants less toxic than wild-type (WT) TDP-43 ( $t$-test false discovery rate, FDR $=0.05)$. The dataset therefore allows us to investigate how mutations both increase and decrease toxicity. Very interestingly, ALS TDP-43 mutations increase toxicity, with a strong bias towards moderate effects $(t$-test, $p$-value $=0.005)$ (Fig. $1 \mathrm{~d}$, Supplementary Fig. $2 \mathrm{~d}$ ).

Mutation effects are largest in a central hotspot of the PRD. Plotting the mean toxicity of all mutations at each position in the sequence reveals a 31 AA hotspot (312-342) where the effects of mutations are strongest (Fig. 1e). The variance in toxicity per position is also the highest within this hotspot, with mutations both strongly increasing and decreasing toxicity (Fig. 1e). A heatmap of the toxicity of all of the single mutations also clearly reveals this hotspot, with most mutations of strong positive or negative effect falling within this 31 AA window (Fig. 1f). Equally strikingly, mutations to the same AA but in different positions within the hotspot often have very similar effects (Fig. 1f). In particular, mutations to charged and polar residues increase toxicity throughout the hotspot and mutations to hydrophobic AAs decrease toxicity (Fig. 1f).

Hydrophobicity and aggregation potential predict toxicity. To more systematically identify features associated with changes in toxicity we made use of all 53,468 variants carrying one or two AA substitutions (excluding STOP codon variants). We used principal components analysis (PCA) to reduce the redundancy in a list of over $350 \mathrm{AA}$ physicochemical properties (Supplementary Fig. 3) and linear regression to quantify how well changes in these physicochemical properties predict changes in the toxicity of TDP-43. A principal component very strongly related to hydrophobicity is the most predictive feature of toxicity, explaining $66 \%$ of the variance in toxicity of all 8,040 mutants within the $312-342$ hotspot and $51 \%$ of the variance in toxicity of all genotypes (Fig. 2a). With the same approach, we tested the performance of established predictors of protein aggregation, intrinsic disorder and other properties. None of them are as predictive as hydrophobicity (Fig. 2b). Importantly, after controlling for hydrophobicity, additional features such as 
a
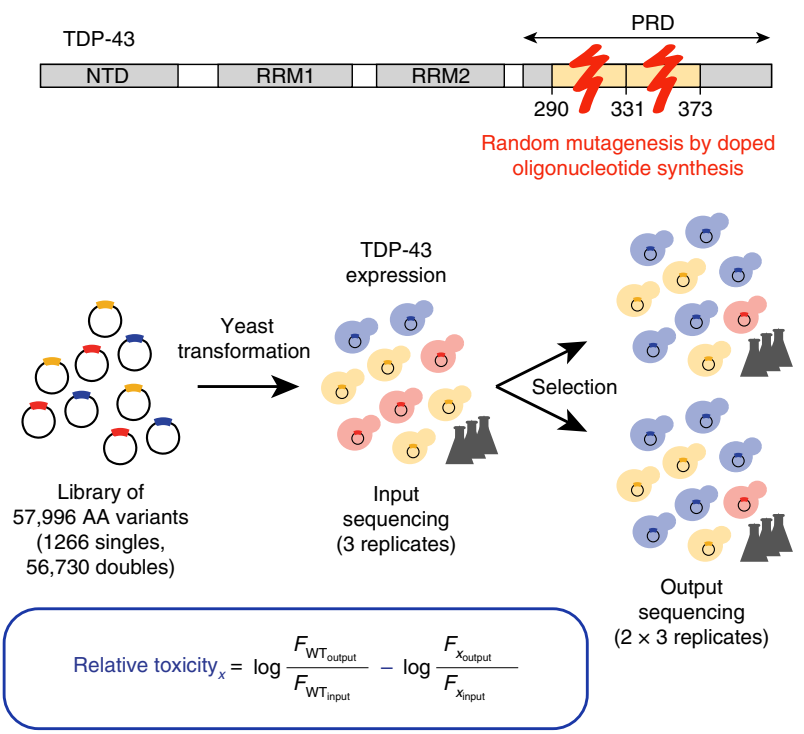

b

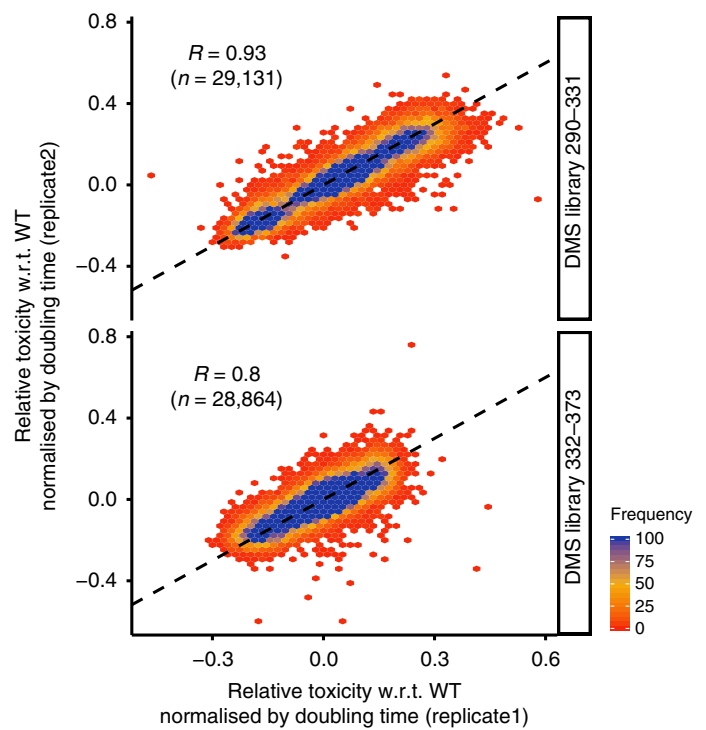

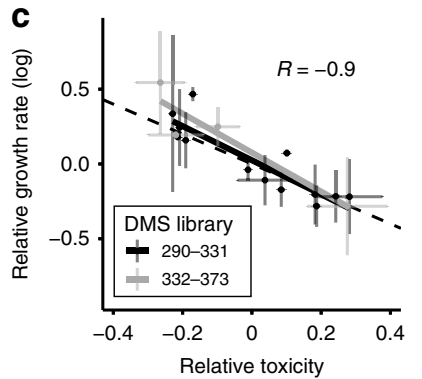

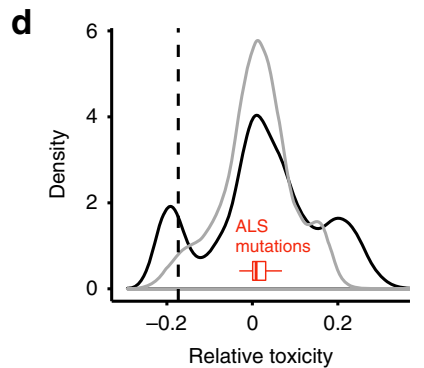

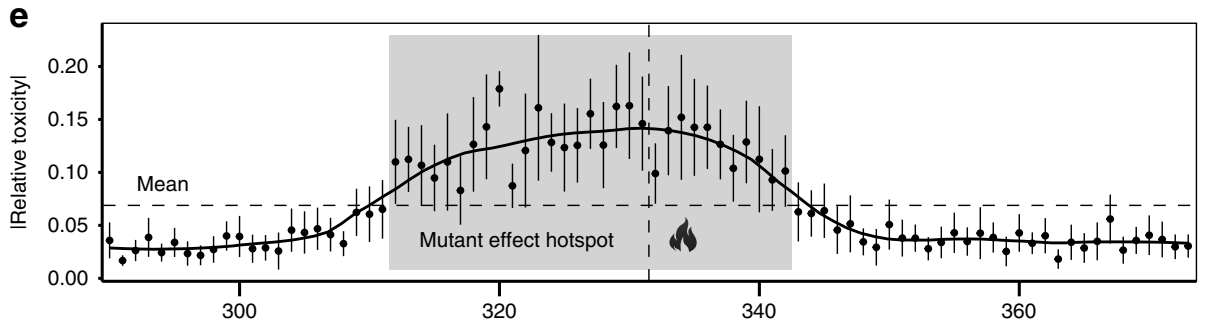

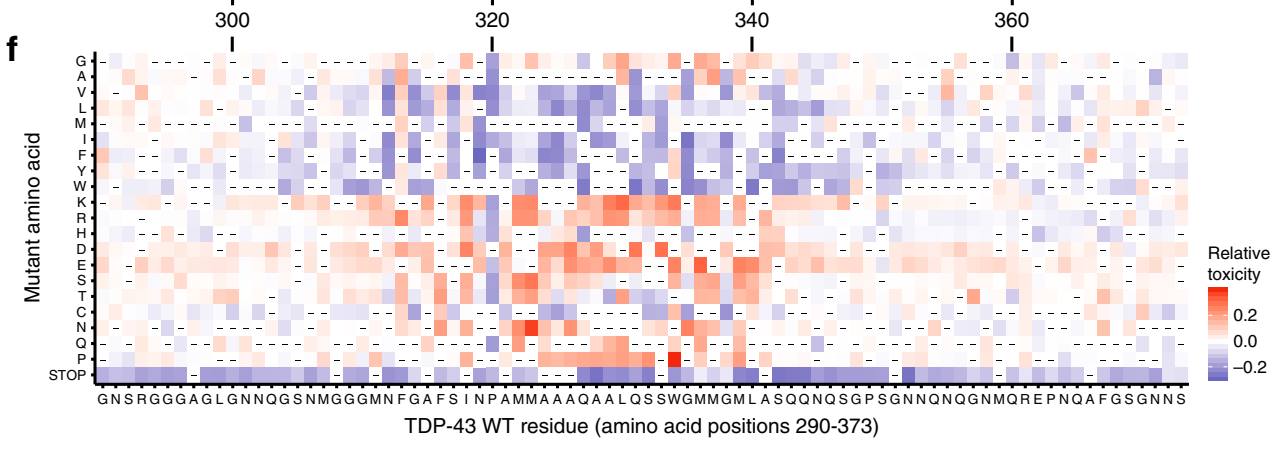

Fig. 1 Deep mutational scanning (DMS) of the prion-like domain (PRD) of TDP-43. a Domain structure of TDP-43 and DMS experimental protocol: For each library, three independent selection experiments were performed. In each experiment one input culture was split into two cultures for selection upon induction of TDP-43 expression ( 6 outputs total). Relative toxicity of variants was calculated from changes of output to input frequencies relative to WT. b Correlation of toxicity estimates between replicates 1 and 2 for single and double amino acid (AA) mutants shown separately for each library (290-332; 332-373). The Pearson correlation coefficients (R) are indicated. Toxicity correlations between all replicates are shown in Supplementary Fig. 1d, e. c Comparison of toxicity from pooled selections and individually measured growth rates for selected variants. Vertical and horizontal error bars indicate 95\% confidence intervals of mean growth rates and toxicity estimates respectively. Linear fits of the data are shown separately for each library and Pearson correlation $(R)$ after pooling data from both libraries is indicated. d Toxicity distribution of single and double mutants, shown separately for each library (colour key as in panel (c)). WT variant has toxicity of zero, mean toxicity of variants with single STOP codon mutation is indicated by dashed vertical line. The red boxplot depicts the distribution of toxicity estimates for all human disease mutations (including sporadic and familial ALS mutations). Outliers are not depicted but are reported in Supplementary Fig. 2d, e. e Absolute toxicity of single mutants stratified by position. Error bars indicate $95 \%$ confidence intervals of mean (per-position) toxicity estimates. A local polynomial regression (loess) over toxicity estimates of all single mutants is shown. The vertical dashed line indicates the boundary between the two DMS libraries. The horizontal dashed line indicates the mean absolute toxicity of all single mutants. The mutant effect "hotspot" (mean per-position |toxicity| > mean |toxicity|) is highlighted in grey. $\mathbf{f}$ Heatmap showing single mutant toxicity estimates. The vertical axis indicates the identity of the substituted (mutant) AA. Heatmap cells of variants not present in the library are denoted by "-"

charge and aromaticity do not predict toxicity (Fig. 2d, e, Supplementary Fig. 4a) with aggregation potential accounting for an additional $4 \%$ of variance in the hotspot (Fig. $2 \mathrm{f}$, g).

That increased hydrophobicity and aggregation potential are strongly associated with reduced toxicity across $>50,000$ genotypes was unexpected given previous work that reported an increased number of intracellular aggregates for a set of TDP-43 variants toxic to yeast ${ }^{54}$ and the widely-held view that aggregation is harmful to cells $s^{42,52,56}$. We therefore further investigated the effects of mutants that alter the hydrophobicity and toxicity of TDP-43. 

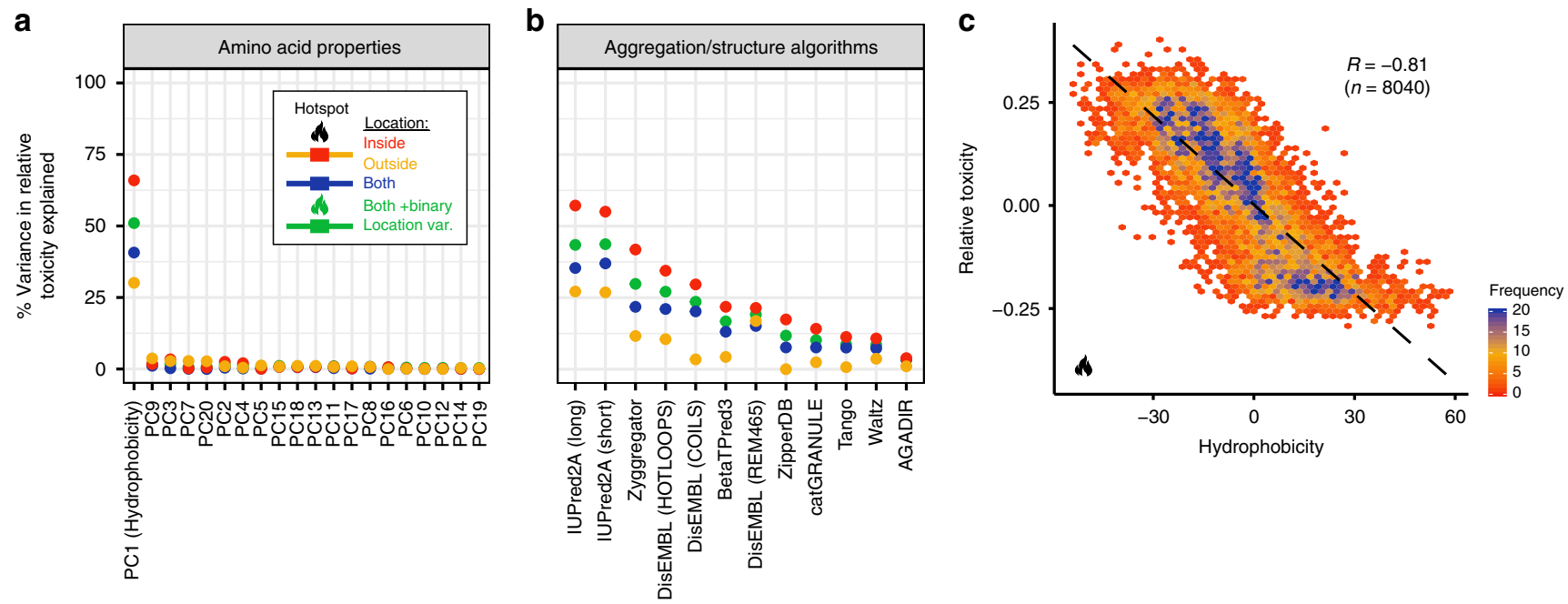

d
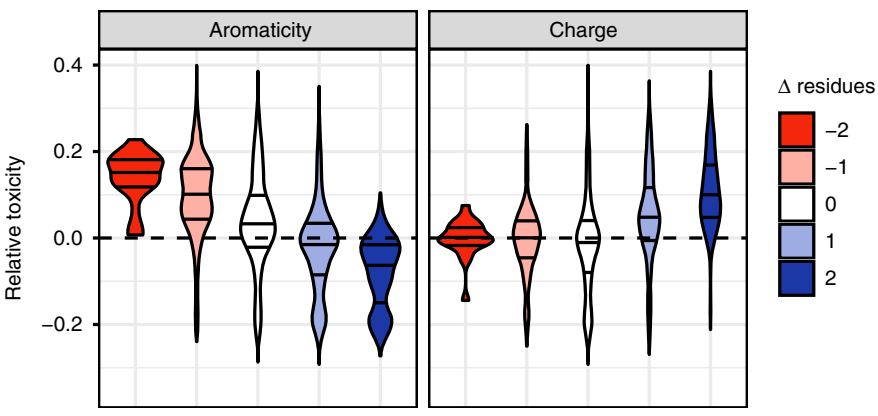

f

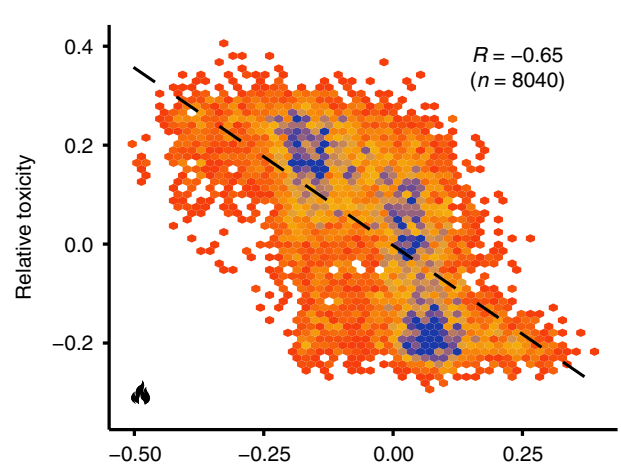

e

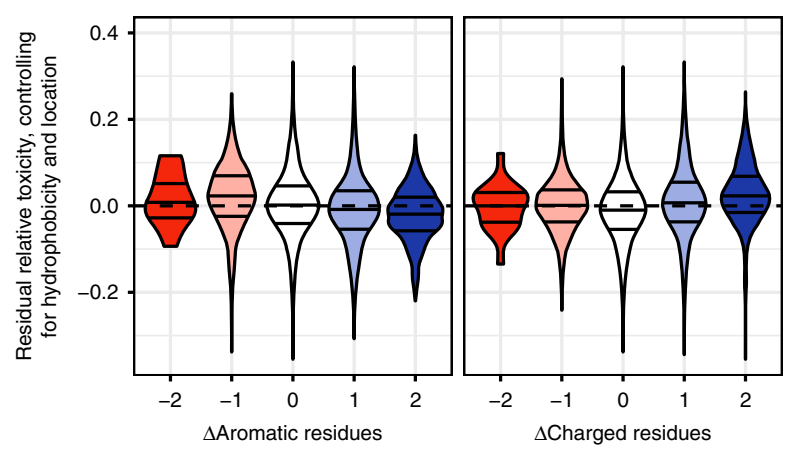

g

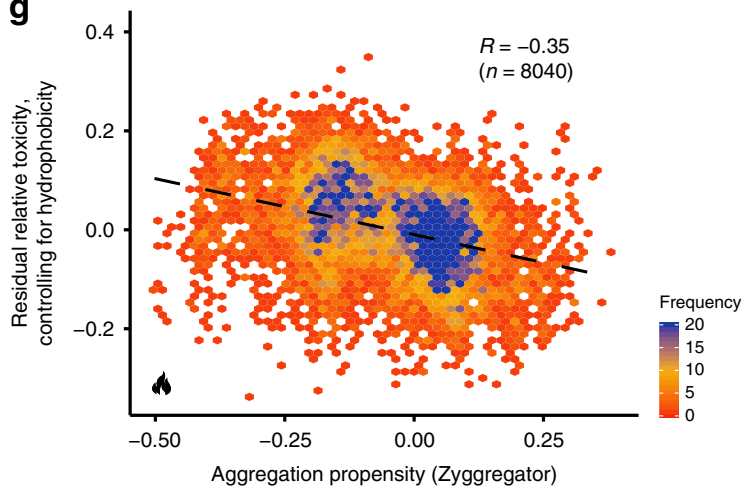

Fig. 2 Changes in hydrophobicity are highly predictive of TDP-43 cellular toxicity. a Percentage variance of toxicity explained by linear regression models predicting single and double mutant variant toxicity from changes in AA properties upon mutation (PCs, principal components of a collection of AA physico-chemical properties). Different regression models were built for different subsets of the data. Simple linear regression models for all variants (blue) or only variants inside (red) or outside (yellow) the hotspot region. And a regression model using all variants and including a binary location variable (inside/outside hotspot) as well as an interaction term between binary location variable and the indicated AA property feature (green). b Percentage variance of toxicity explained by linear regression models predicting variant toxicity using scores from aggregation/structure algorithms (see Methods). Colour key shown in panel (a). See also Supplementary Fig. 4. c Toxicity of variants with single or double mutations within the hotspot region as a function of hydrophobicity changes (PC1) induced by mutation. The Pearson correlation (R) before binning is indicated. See also Supplementary Fig. 9a. d Toxicity distributions of single and double mutants stratified by the change in the number of aromatic $(\mathrm{H}, \mathrm{F}, \mathrm{W}, \mathrm{Y}, \mathrm{V})$ or charged residues $(R, D, E, K)$ relative to the WT sequence. Horizontal axis as in panel (e). e Distribution of residual toxicity after controlling for the effect of hydrophobicity and location on toxicity (green regression model in panel a) stratified by the number of aromatic $(H, F, W, Y, V)$ or charged (R,D,E,K) $A A s$. f Single and double mutant variant toxicity as a function of changes in aggregation propensity (Zyggregator). Only variants occurring within the toxicity hotspot are depicted. The Pearson correlation (R) before binning is indicated. $\mathbf{g}$ Toxicity as a function of aggregation propensity after controlling for hydrophobicity (red regression model in panel a). Only variants occurring within the toxicity hotspot are depicted. The Pearson correlation ( $R$ ) before binning is indicated. See also Supplementary Fig. 9b 
a
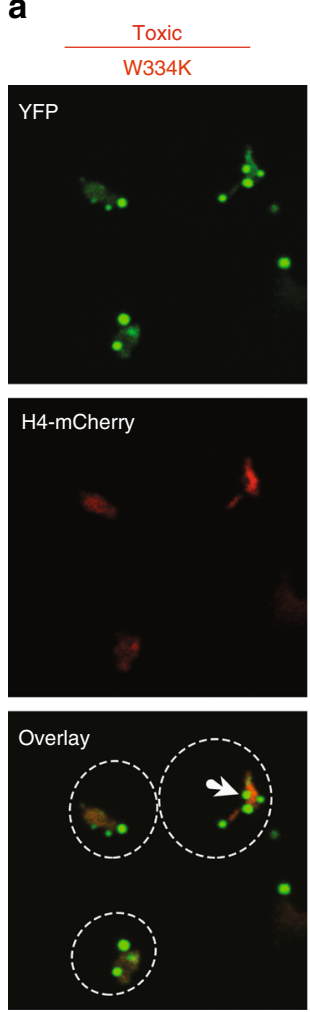
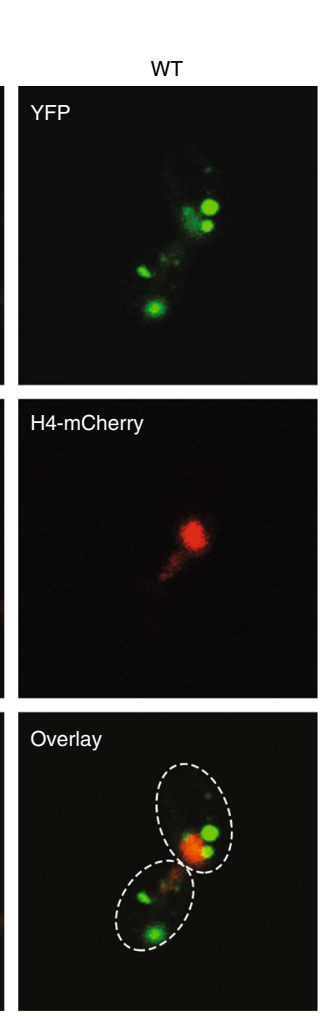
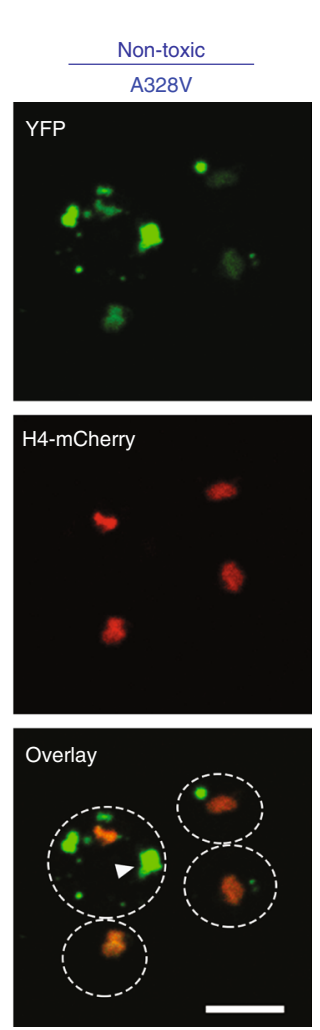

b
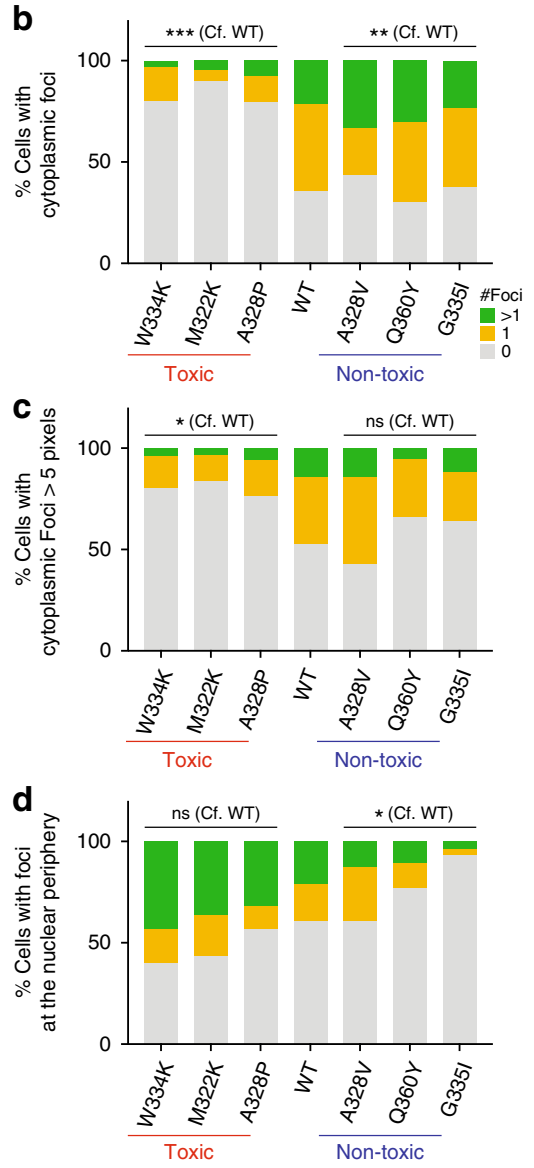
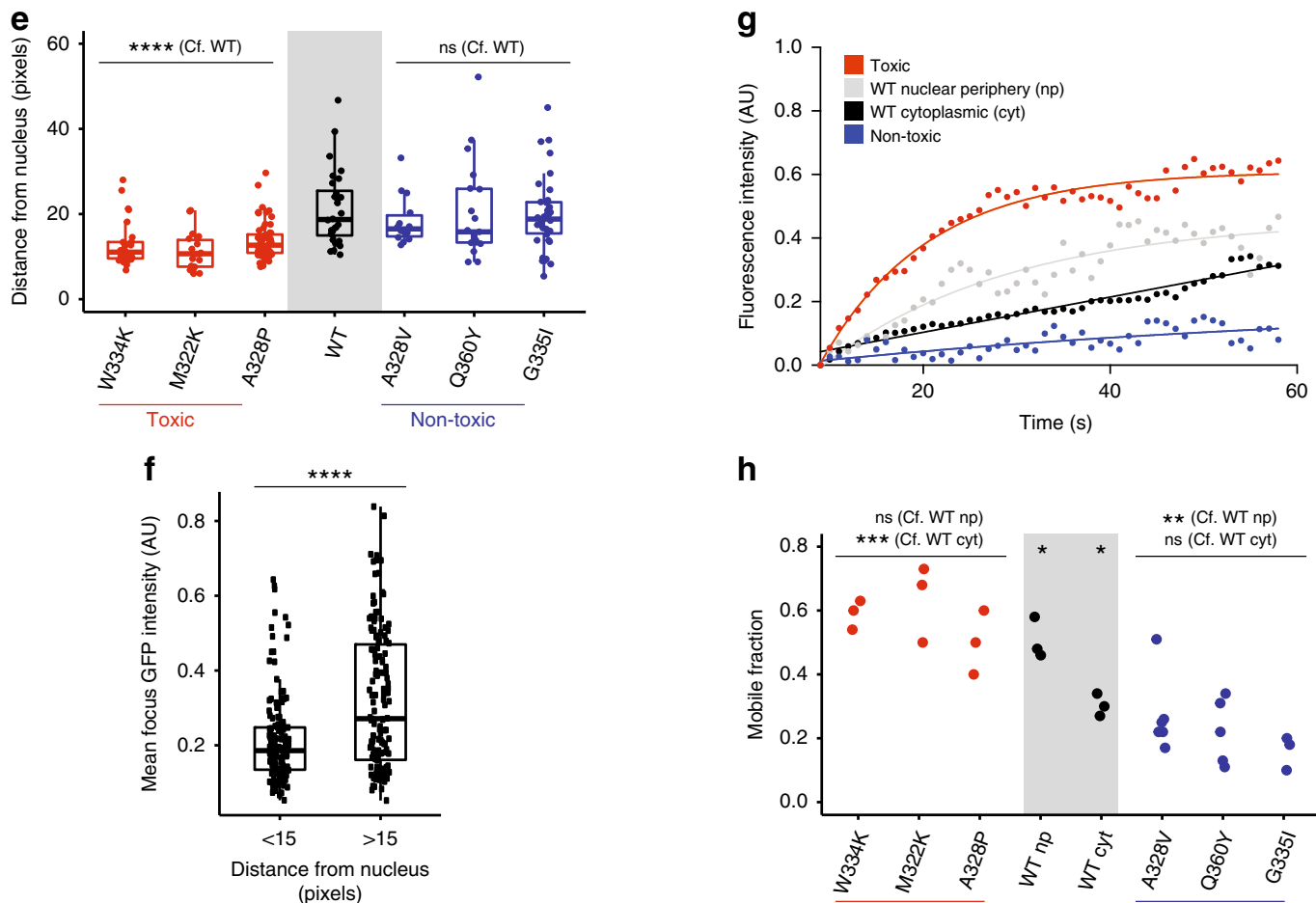

h

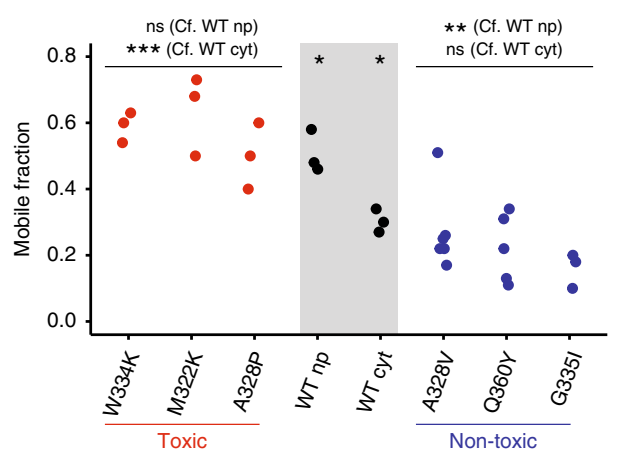

Two classes of cytoplasmic TDP-43 foci. WT TDP-43 localizes to both the nucleus and to the cytoplasm of yeast cells $s^{54,55}$ (Fig. 3a). In the nucleus, TDP-43 is diffuse, but in the cytoplasm it forms puncta, consistent with previous observations ${ }^{41,57}$. We observe that cytoplasmic WT TDP-43 forms two types of assemblies: small foci in the nuclear periphery and larger foci detached from the nucleus (Fig. 3a, c). We find that mutations that decrease TDP-43 hydrophobicity and increase TDP-43 toxicity increase the number of the small foci at the nuclear periphery and reduce the number of large distal foci (Fig. 3b, c, f, 
Fig. 3 Mutations leading to formation of solid-like aggregates rescue toxicity. a Representative fluorescence microscopy images of yeast cells expressing indicated YFP-tagged TDP-43 variants (W334K TDP-43 = toxic, A328V TDP-43 = non-toxic). H4-mCherry marks nuclei (red). Contrast was enhanced equally for the green and red channels in all images. $\mathbf{b}$ Percentage of cells with cytoplasmic foci (Cells scored: $n[$ toxic $]=219, n[$ WT $]=30, n[$ non-toxic $]=$ 213). Fisher's Exact test. c Percentage of cells with cytoplasmic foci with size over 5 pixels automatically detected by CellProfiler. Fisher's Exact test. (Cells scored: $n[$ toxic $]=167, n[\mathrm{WT}]=23, n$ [non-toxic] $=167$ ). $\mathbf{d}$ Percentage of cells with foci at the nuclear periphery (Cells scored: $n$ [toxic] $=219, n[\mathrm{WT}]=$ 30, $n$ [non-toxic] $=213$ ). Fisher's exact test. e Distance of foci from nucleus center for toxic (red), non-toxic (blue), and WT (black) TDP-43. Boxplots represent median values, interquartile ranges and Tukey whiskers with individual data points superimposed. Kruskal Wallis with Dunn's multiple comparisons test ( $n=>20 \mathrm{foci} /$ variant). f Average fluorescence intensity of foci localized closer ( $<15$ pixels, $n=147$ ) or further ( $>15$ pixels, $n=138)$ from the nucleus. Boxplots represent median values, interquartile ranges and Tukey whiskers with individual data points superimposed. Mann-Whitney test. g Representative individual fluorescence recovery traces for variants reported in panel (e). Lines are the result of a single exponential fitting. $\mathbf{h}$ Mobile Fraction as calculated by fitting FRAP traces for toxic (red), non-toxic (blue) and WT (black) TDP-43. Each point results from fitting an individual trace. One-way ANOVA with Tukey's multiple comparisons test. Images were taken on cells growing from at least 3 independent starting colonies. ${ }^{\star} P<0.05$, ${ }^{\star \star} P<0.01,{ }^{\star \star \star} P<0.001,{ }^{\star \star \star \star} P<0.0001$. Scale bar $=5 \mu \mathrm{M}$. Source data are provided as a Source Data file

Supplementary Fig. 5a). TDP-43 mutations reported in ALS (Supplementary Fig. 2e) also increase the number of foci at the nuclear periphery compared to WT TDP-43 (Supplementary Fig. $6 \mathrm{a}, \mathrm{b})$. In contrast, mutations that increase hydrophobicity and reduce toxicity reduce the number of small nucleusassociated foci and increase the number of large distal foci (Fig. 3b, c, f, Supplementary Fig. 5a).

Toxic mutations promote dynamic liquid-like condensates. We used fluorescence recovery after photobleaching (FRAP) to characterize the dynamics of TDP-43 variants in the different foci. The large cytoplasmic foci formed by non-toxic variants show little exchange of TDP-43 molecules with the soluble cytoplasmic pool. In contrast, the small foci localized at the nuclear periphery can exchange more protein with the cytoplasm, consistent with a more liquid-like state (Fig. 3d, e). Such differences in dynamics have been described also for distinct types of misfolded protein compartments ${ }^{58}$. Both types of compartments co-localize with the yeast chaperone Hsp104 (Supplementary Fig. 7a). The large immobile TDP-43 foci are also brighter than the small dynamic ones (Fig. 3g), similar to what has been observed for Huntingtin variants that partition between immobile bright assemblies and liquid-like dimmer ones ${ }^{59}$. The non-toxic TDP-43 variants also have a higher protein concentration quantified by Western blotting (Supplementary Fig. 5b).

Taken together, these results suggest that mutations that increase the hydrophobicity of TDP-43 result in a re-localization of the protein away from small and dynamic, liquid-like foci at the nuclear periphery to large and more solid aggregates in the cytoplasm. A reduction in hydrophobicity has the opposite effect.

Genetic interactions reveal the hotspot structure in vivo. The hotspot region of the TDP-43 PRD (AA 312-342) is a conserved region $^{35,36}$, with hydrophobicity more similar to the globular domains of TDP-43 than to the surrounding hydrophilic disordered regions (Fig. 4b). The hotspot is contained within a region (311-360) that was previously shown to be sufficient for both in vitro aggregation and the formation of cytoplasmic foci ${ }^{35}$. Fragments from within this region have previously been shown to have the potential to form different types of secondary structures in vitro. More specifically, nuclear magnetic resonance (NMR) spectroscopy of the PRD revealed that residues 321-342 can adopt an $\alpha$-helical structure in certain conditions $s^{35,36,47}$ and four different 6-11 AA peptides from the region could form cross- $\beta$ amyloid or amyloid-like fibrils whose structures were determined by X-ray crystallography ${ }^{52}$. However, it is unknown whether any of these structures exist in vivo for full-length TDP-43.

We have shown recently that the pattern of genetic (epistatic) interactions between mutations in a protein can report on the secondary structure of that molecule when it is performing the function that is being selected for ${ }^{51,60}$. In particular, when a sequence forms an $\alpha$-helix, the side chains of residues separated by 3-4 AA are close in space and similarly oriented so that mutations in these AA interact similarly with mutations in the rest of the protein. In contrast, in a $\beta$-strand, the side chains of residues separated by $2 \mathrm{AA}$ are close and similarly oriented and so make similar genetic interactions with other mutations (Fig. $4 \mathrm{a})^{61}$.

We used the 52,272 double mutants (excluding STOP codon variants) in our dataset to identify pairs of mutations that genetically interact. We first identified pairs of mutations that had unexpectedly high or low toxicity $(<5$ th and $>95$ th percentile of the expected toxicity distribution, negative and positive epistasis for growth rate, respectively). We then quantified the similarity of epistasis enrichment profiles between pairs of positions and compared these patterns to those expected for $\alpha$-helices and $\beta$ strands, scoring significance by randomization ${ }^{51}$ (Fig. 4a).

This revealed that the patterns of epistasis in our dataset are consistent with two secondary structure elements forming inside the PRD in vivo: a $\beta$-strand at residues $311-316$ and an $\alpha$-helix at residues $324-331$ (Fig. 4c). The $\beta$-strand identified by the epistasis analysis coincides with one of the peptides in the TDP-43 PRD that, in vitro, can form cross- $\beta$ structures ${ }^{52}$ typical of protein aggregates (Fig. 4d). The crystals of this specific peptide consist of a non-conventional $\beta$-strand termed a low-complexity aromaticrich kinked segment (LARKS $)^{62}$. In this in vitro structure, Phe 313 and Phe 316 face the same side of the sheet, whereas in a canonical sheet the side chains of odd and even residues face opposite sides. Strikingly, this non-canonical contact between Phe 313 and Phe 316 is also identified by the in vivo epistasis analysis, with a similarity in interaction profile ranking amongst the top two residue pairs in this region. In addition, the contact between Phe 316 and Ala 315, which again is compatible with a LARKS but not with a canonical $\beta$-strand has the highest predicted contact score among neighbouring residues (Fig. 4d). The predicted contact map built on the basis of in vivo epistatic interactions strikingly matches the Protein Data Bank (PDB) structure for LARKS 312-317 (Fig. 4d, Supplementary Fig. 8).

On the other hand, the genetic interactions of mutations in the 324-330 region match those expected for an $\alpha$-helix (Fig. 4e). This region is part of the portion (321-342) of TDP-43 that can transiently and cooperatively fold into an $\alpha$-helix in vitro $36,47,63$. This helix is stabilized by inter-molecular contacts and its selfinteraction was proposed to seed liquid-demixing in vitro. Amyloid fibrils can grow from the liquid de-mixed state and circular dichroism spectroscopy revealed that the helix can transition to a $\beta$-sheet over time, compatible with the process of aggregation $^{35,63}$. On the basis of epistasis, the top scoring predicted contacts in this region are between residues separated 
a
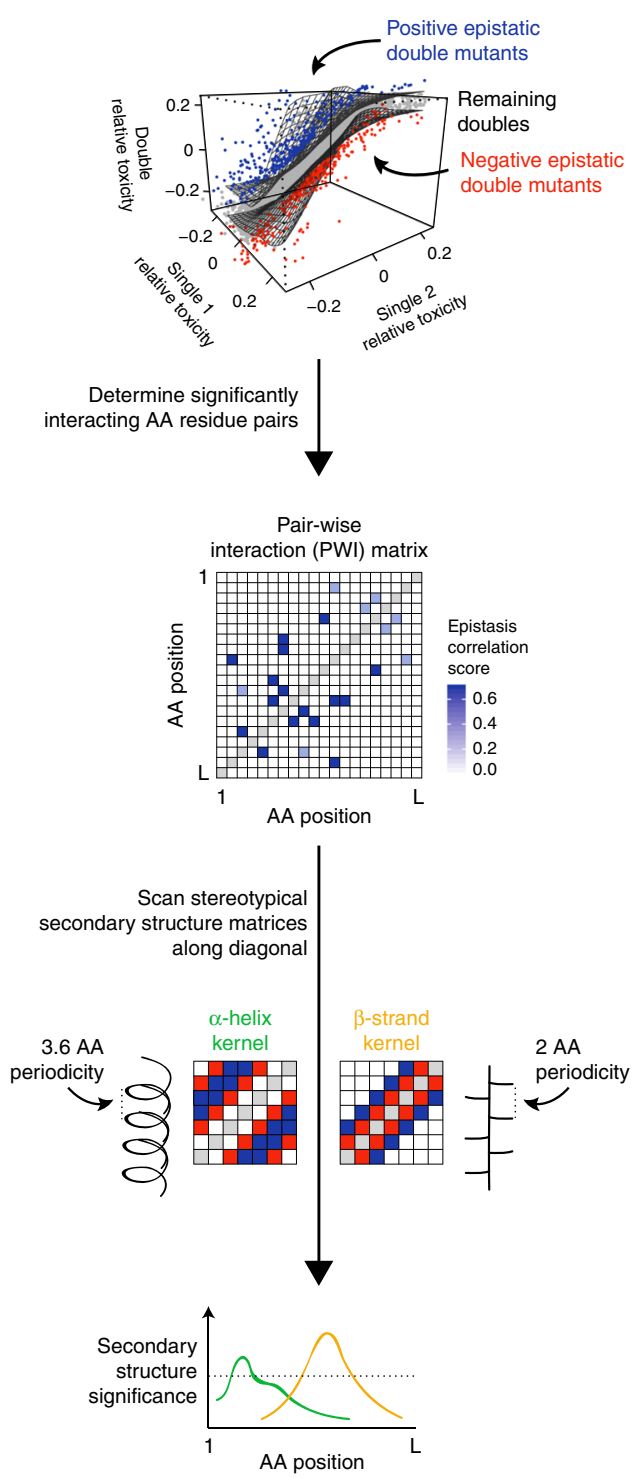

b
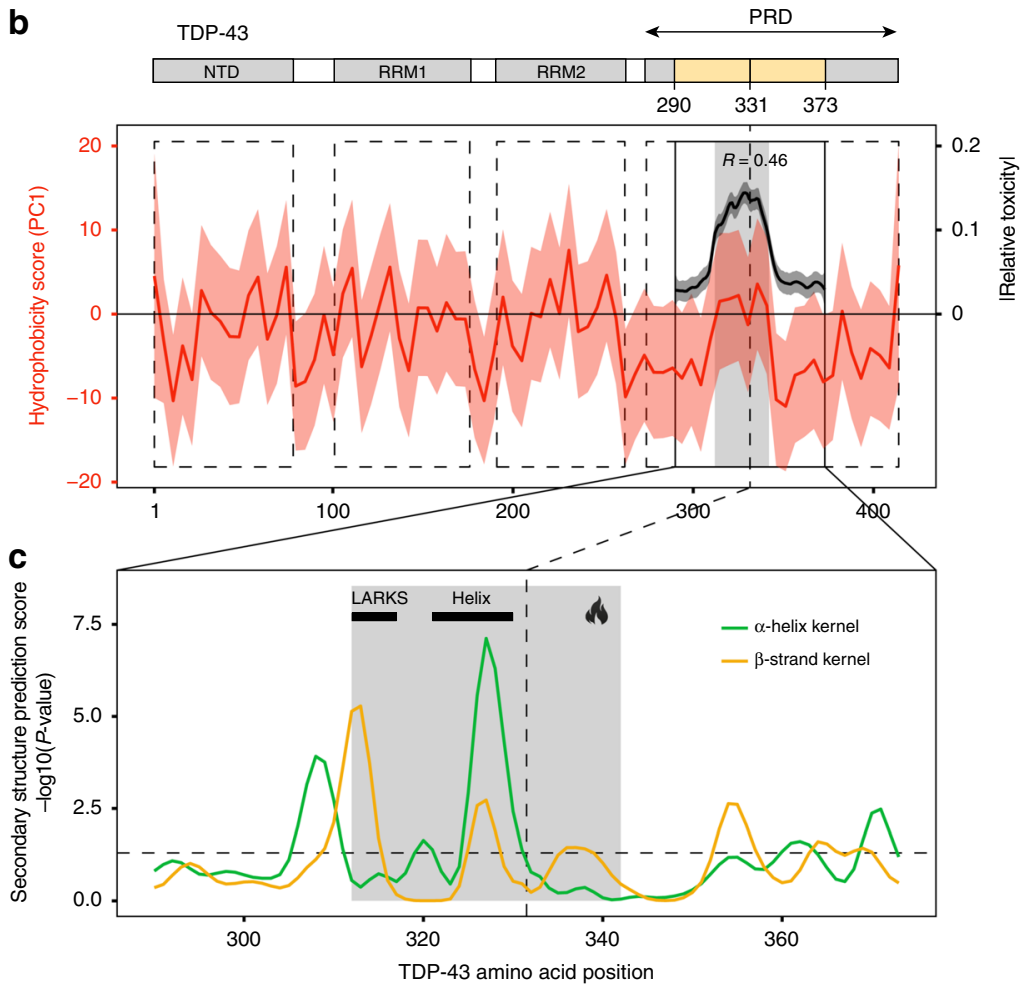

d

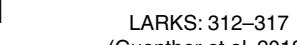

e (Guenther et al. 2018)
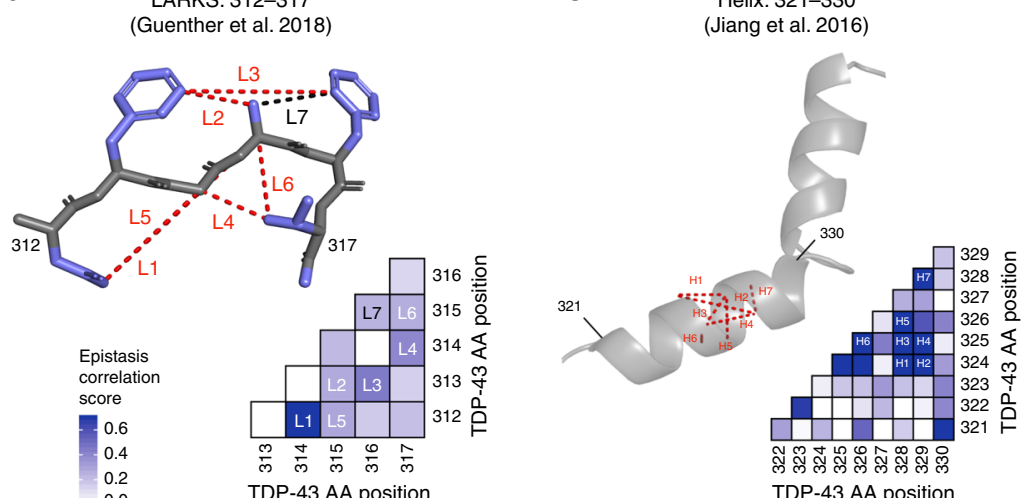

Fig. 4 Correlated patterns of epistasis predict secondary structural elements within the PRD of TDP-43. a Schematic representation of the computational strategy to identify in vivo secondary structures. Double mutant variants are classified as epistatic if they are more (95th percentile) or less (5th percentile) toxic than other variants with similar single mutant toxicities (top). A pair-wise interaction (PWI) matrix of epistasis correlation scores is then constructed by quantifying the similarity of a pair of positions' interactions with all other mutated positions in the protein. The epistasis correlation scores along the diagonal of the PWI matrix are then tested for agreement with the stereotypical periodicity of $\alpha$-helix and $\beta$-strand, using two-dimensional kernels (bottom), to calculate the likelihood of adjacent positions forming secondary structures. b Local polynomial regression (loess) of hydrophobicity (PC1) of the WT TDP-43 sequence with 95\% confidence interval. For reference, smoothed toxicity estimates in the mutated positions within the PRD are shown. The Pearson correlation coefficient $(R)$ between hydrophobicity and mean toxicity effects of single mutants at each position before smoothing is indicated. c Secondary structure predictions from epistasis correlation scores for $\alpha$-helix and $\beta$-strand kernels based on the strategy described in panel a. Black bars annotate previously described structural features: LARKS, low-complexity aromatic-rich kinked segment $(312-317)^{52}$; Helix $(321-330)^{35}$. The dashed horizontal line indicates the nominal significance threshold $P=0.05$. d Epistatic interactions in region 312-317 are consistent with positions of similar side-chain orientations interacting in a previously reported in vitro LARKS structure. Epistasis correlation matrix and top seven epistasis correlation score interactions annotated on the LARKS reference structure (monomer from PDB entry 5whn, https://www.rcsb.org/structure/5WHN). Dashed lines on structure connect interacting residues at minimal distance between side chain heavy atoms. Side chain atoms are depicted in blue. e Epistatic interactions in region 321-330 are consistent with positions of similar side-chain orientations interacting in an $\alpha$-helix. Epistasis correlation matrix and top seven epistasis correlation score interactions annotated on the Helix reference structure (monomer from PDB entry 5whn, https://www.rcsb.org/structure/5WHN)

by $3-4$ AA such as Ala 324 and Ala 328, or Ala 325 and Ala 328, consistent with interactions between side chains of an a-helix (Fig. 4e).
The pattern of in vivo epistatic interactions between mutations in TDP-43 therefore is compatible with a model in which two of the secondary structures that have previously been observed 
in vitro for fragments of TDP-43 actually form in vivo in the fulllength protein.

\section{Discussion}

Specific protein aggregates have long been recognized as the hallmarks of many neurodegenerative diseases ${ }^{4-6,52,64}$. However, whether these aggregates are the cause of these diseases, nonpathological by-products, or a protective mechanism is still very unclear and hotly debated ${ }^{13-16}$. Indeed, although it is often assumed to be the case, it is not even clear whether aggregates are the cause of toxicity when aggregating proteins are expressed in simpler cellular systems ${ }^{54,55}$. We reasoned that deep mutagenesis might be an effective approach to resolve this question.

In this study, we have tested this approach using the ALS protein TDP-43 that both aggregates and causes toxicity in the model eukaryote, S. cerevisiae. Quantifying the effects of $>50,000$ mutants of TDP-43 revealed unequivocally that increasing the hydrophobicity and aggregation of TDP-43 strongly reduces the toxicity of this protein in yeast cells. Consistently, mutations that reduce hydrophobicity and the aggregation potential of TDP-43 increase the toxicity of the protein. Although they reduced the formation of large, solid aggregates, mutations that increase toxicity promote the formation of alternative foci-dynamic, liquid-like TDP-43 condensates clustered at the nuclear periphery. We propose therefore that aggregation reduces the toxicity of TDP-43 to yeast cells because it titrates TDP-43 away from this toxic liquid-like phase (Fig. 5a).

a
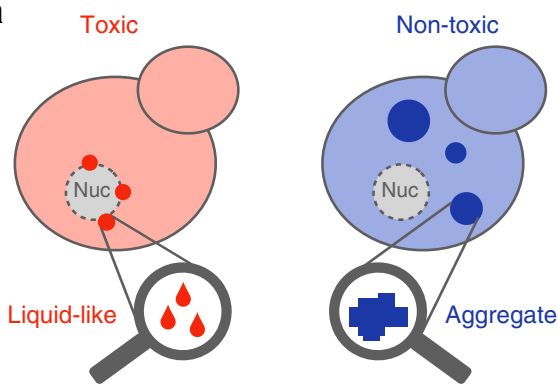

b

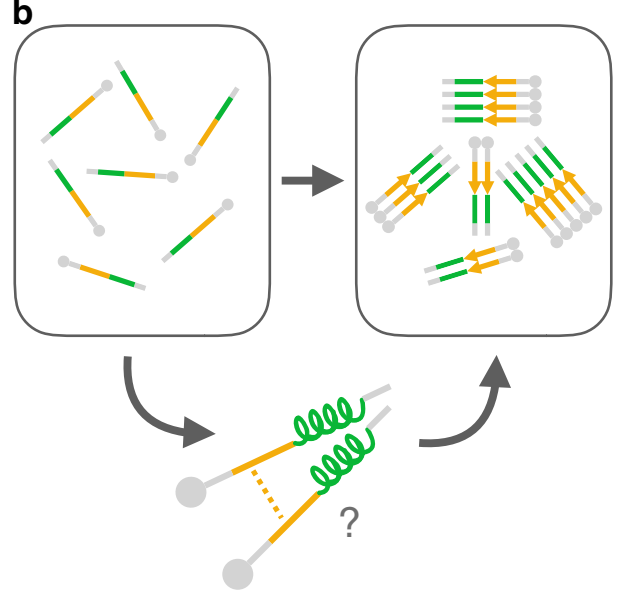

Fig. 5 Model of how AA changes determine toxicity of TDP-43. a Mutations that promote formation of insoluble cytoplasmic aggregates decrease TDP-43 toxicity, while mutations that cause the protein to stall in a liquid de-mixed phase increase its toxicity to the cell. $\mathbf{b}$ Secondary structure elements, within the toxicity hotspot 312-342, promote the aggregation process of TDP-43, with a transient helix forming on pathway to $\beta$-rich aggregates
That TDP-43 aggregates are protective rather than toxic is consistent with previous work in multiple systems, including the rescue of toxicity by the accumulation of RNA lariats that sequester TDP-43 into large aggregates ${ }^{65}$. Moreover, in mammalian cells, liquid de-mixed TDP-43 was recently shown to recruit the nuclear pore component Nup62 and the importin- $\alpha$ transporter, resulting in nuclear transport impairment and toxicity ${ }^{44}$. Thus, although it still remains to be established whether aggregation of TDP-43 is also protective in mammalian cells and neurons, it seems likely that this will be the case. The observation that all recurrent fALS mutations increase the toxicity of TDP-43 in yeast and by a similar magnitude (Supplementary Fig. 2d) is very striking and suggests that the yeast system may indeed capture molecular mechanisms relevant to the human disease. Indeed, given the late age of onset of ALS, it is particularly interesting that the fALS mutations are all moderate effect mutations when expressed in yeast, as it may be the case that the more toxic variants of TDP-43 are embryonic lethal in humans.

More generally, our results demonstrate that deep mutagenesis is a powerful approach for determining the sequence-function relationships of intrinsically disordered proteins, including probing their in vivo structures. Mutations had their strongest effects within a central hotspot region of the TDP-43 PRD. Our recently developed approach ${ }^{51}$ that uses the patterns of genetic interactions in double mutants to report on structural contacts reveals that this 'unstructured' hotspot region is very likely to be structured in vivo with the formation of these secondary structures altering the toxicity of the protein. Indeed, secondary structure elements within this region have been shown to be important for the phase separation and aggregation of fragments of TDP-43 in vitro $35,36,52$. A parsimonious model based on previous in vitro work $35,36,47$ is that the helix forms first in the pathway of aggregation towards a $\beta$-rich species (Fig. 5b). Consistent with this, destabilizing mutations, such as any substitution of Phe 313 and Phe 316 in the LARKS, or the introduction of proline into the 324-330 helix, increase toxicity (Fig. 1f).

The conformations of 'unstructured' proteins are notoriously difficult to study and the interactions between mutations in double mutants provide a general method to probe the in vivo structures of these proteins whenever a selection assay is available. We envisage that this approach can be adopted to study the functions, toxicity, and in vivo structures of other intrinsically disordered proteins, including the many other proteins implicated in neurodegenerative diseases.

Our conclusions derived from deep mutagenesis of TDP-43 are also consistent with observations for other genes, such as the reduced toxicity of SOD-1 variants that increase aggregation ${ }^{16,66}$ and the increased survival of neurons containing Huntingtin inclusion bodies ${ }^{67}$. They are also consistent with increasing evidence that insoluble aggregates are not pathogenic in multiple other neurodegenerative diseases $64,68,69$, and with the clinical failure of therapeutic approaches that reduce the occurrence of aggregates $10,12,70-72$.

Indeed, if insoluble aggregates generally titrate proteins away from alternative toxic phases, interactions and functions, then promoting rather than alleviating aggregation might be the more appropriate therapeutic goal in neurodegenerative diseases.

\section{Methods}

Yeast strains and plasmids. Saccharomyces cerevisiae S288C BY4741 (MATa his $3 \Delta 1$ leu $2 \Delta 0$ met $15 \Delta 0$ ura3 $\Delta 0$ ) was used in all experiments. Plasmid pRS416 containing TDP-43 or TDP-43-YFP under control of the Gall promoter was purchased from Addgene ${ }^{54}$. Mutagenesis for the characterization of TDP-43 variants was performed through PCR linearization with specifically-designed primers (Supplementary Data 1, primers: BB_1 to BB_6). The resulting products were then either treated with DpnI or purified from a $1 \%$ agarose gel with a QIAquick Gel Extraction Kit (Qiagen) and transformed into E.coli DH5a competent cells 
(Invitrogen) for plasmid purification and validation through Sanger sequencing. The plasmid used in the co-localization assays contains RNQ1-mCherry under control of the Gall promoter was a kind gift from the Rick Gardner lab. Genes coding for the other proteins for which co-localization was tested were cloned in this plasmid by gap-repair.

Library construction. Two $186 \mathrm{nt}$ oligonucleotides were purchased from TriLink. Each consisted of a 'doped' region of $126 \mathrm{nt}$, corresponding to TDP-43 AA 290-331 or AA 332-373, flanked by $30 \mathrm{nt}$ of the WT TDP-43 sequence on each side. Each position in the mutated area, was doped with an error rate of $1.59 \%$. The target frequency for each library was $27.0 \%$ for single mutants and $27.3 \%$ for double mutants. With this approach, the WT sequence was represented with a frequency of $13.3 \%$. Although a barcoding strategy ${ }^{73}$ could have improved the robustness of sequencing reads, we estimated that the impact of misreads due to the direct sequencing approach here employed would sum up to less than two additional counts per double nucleotide variant attributable to sequencing error (see Variants Toxicity and Error Estimates). Each oligonucleotide was amplified by PCR (Q5 High-Fidelity DNA Polymerase, NEB) for 15 cycles, purified using an Egel electrophoresis system (Agarose 2\%) followed by column purification with a MinElute PCR Purification Kit (Qiagen). In order to introduce the doped sequence in the full-length TDP-43 sequence, the purified oligonucleotide was cloned into $100 \mathrm{ng}$ of linearized pRS416 Gal TDP-43 by a Gibson approach (Supplementary Data 1, primers BB 7 to BB 10). The product was then transformed into 10-beta Electrocompetent E. coli (NEB), by electroporation in a Bio-Rad GenePulser machine $(2.0 \mathrm{kV}, 200 \Omega, 25 \mu \mathrm{F})$. Cells were recovered in SOC medium (NEB) for $30 \mathrm{~min}$ and plated on LB with ampicillin. A total of $\sim 2.7 \times 10^{6}$ transformants were estimated. The plasmid library was purified with a GeneJET Plasmid Midiprep Kit (Thermo Scientific)

Yeast transformation and selection experiments. Yeast cells were transformed with the TDP-43 doped plasmid in 4 independent biological replicates for each library. One single colony was grown overnight in $30 \mathrm{ml}$ YPDA medium at $30^{\circ} \mathrm{C}$ for each replica. Cells were diluted to 0.3 optical density at a wavelength of $600 \mathrm{~nm}$ (OD600) in $175 \mathrm{ml}$ of YPDA and incubated for $4 \mathrm{~h}$ at $30^{\circ} \mathrm{C}$. Cells were then harvested, washed, re-suspended in $8.575 \mathrm{~mL}$ SORB $(100 \mathrm{mM} \mathrm{LiOAc}, 10 \mathrm{mM}$ Tris $\mathrm{pH} 8.0,1 \mathrm{mM}$ EDTA, $1 \mathrm{M}$ sorbitol) and incubated for $30 \mathrm{~min}$ at room temperature. For the transformation, $10 \mathrm{mg}$ per $\mathrm{mL}$ of salmon sperm DNA and $3.5 \mu \mathrm{g}$ TDP-43 plasmid library were used. Cells were mixed to $100 \mathrm{mM} \mathrm{LiOAc}, 10 \mathrm{mM}$ Tris- $\mathrm{HCl}$ pH 8.0, $1 \mathrm{mM}$ EDTA/NaOH pH 8.0 and 40\% PEG 3350. Heat-shock was performed for $20 \mathrm{~min}$ at $42^{\circ} \mathrm{C}$. YPD with $0.5 \mathrm{M}$ sorbitol was used to recover the cells, incubating them for $1 \mathrm{~h}$ at $30^{\circ} \mathrm{C}$. After recovery, cells were resuspended in SC-URA $2 \%$ raffinose medium, while an aliquote was plated to calculate transformation efficiency.

After $\sim 50 \mathrm{~h}$ of growth, cells were diluted in SC-URA $2 \%$ raffinose medium and grown for 4.5 generations. At this stage, $400 \mathrm{~mL}$ of each replica were harvested, washed, split into two tubes and frozen at $-20^{\circ} \mathrm{C}$ for later extraction of input DNA. To induce plasmid expression, for each replicate two cultures were diluted in SC-URA $2 \%$ galactose medium. After 5-6 generations, $2 \times 400 \mathrm{~mL}$ for each replicate were harvested to obtain output pellets for DNA extraction.

DNA extraction and library preparation. Input and Output pellets were resuspended in $1.5 \mathrm{~mL}$ extraction buffer ( $2 \%$ Triton-X, $1 \%$ SDS, $100 \mathrm{mM} \mathrm{NaCl}, 10 \mathrm{mM}$ Tris-HCl pH 8.0, 1 mM EDTA pH 8.0). Two cycles of freezing in an ethanol-ice bath and heating at $62{ }^{\circ} \mathrm{C}$ were performed. Deproteinization was performed using 25:24:1 phenol-chloroform-isoamyl alcohol and glass beads. After centrifugation, the aqueous phase, containing the DNA, was recovered and treated again with phenol-chloroform-isoamyl alcohol. The samples were incubated for $30 \mathrm{~min}$ at $-20^{\circ} \mathrm{C}$ with $1: 10 \mathrm{~V} 3 \mathrm{M} \mathrm{NaOAc}$ and $2.2 \mathrm{~V} 100 \%$ ethanol for DNA precipitation. At this stage and after centrifugation for $30 \mathrm{~min}$, the pellets were dried overnight at room temperature. RNA was eliminated by incubation with RNAse $10 \mathrm{mg}$ per $\mathrm{mL}$ for $30 \mathrm{~min}$ at $37^{\circ} \mathrm{C}$. DNA purification was achieved with a QIAEX II Gel Extraction Kit (Qiagen) and DNA was eluted in $375 \mu \mathrm{L}$ of elution buffer. DNA concentration was measured by q-PCR, with primers annealing to the Ori site of the pRS416 plasmid (Supplementary Data 1, primers BB_11, BB_12).

The TDP-43 library was then prepared for deep sequencing by PCR amplification in two steps using Q5 High-Fidelity DNA Polymerase (NEB). In step 1,300 million plasmids were amplified for 15 cycles using frame-shifted adaptor primers with partial homology to standard Illumina sequencing primers (Supplementary Data 1, primers BB_13 to BB_47). Samples were treated with ExoSAP (Affymetrix) and purified with QIAEX II kit (Qiagen). PCR products from the first step were used as templates in the second PCR step, where indexed Illumina primers (Supplementary Data 1, primers TS_HT_D7X_7 to

TS_HT_D7X_95) were used for a 10 cycles amplification. DNA concentration was then quantified by means of a Quant-iT ${ }^{\text {Tw }}$ PicoGreen ${ }^{\circledast}$ dsDNA Assay Kit (Promega). All replicates were pooled together in an equimolar ratio. Finally, the pooled sequencing library was run on a $2 \%$ agarose gel, purified and sent for 125 base-pair (bp) paired-end Illumina sequencing at the CRG Genomics Unit.
Individual growth rate measurements. Yeast cells expressing selected TDP-43 variants were grown overnight in SC-URA $2 \%$ raffinose non-inducing medium and diluted to 0.2 OD600 until exponential phase. Then they were diluted to 0.1 OD600 in SC-URA $2 \%$ galactose to assess growth in inducing conditions. Growth was monitored by measuring OD600 in a 96-well plate at 10 min intervals inside an Infinite M200 PRO microplate reader (Tecan). Plates were kept constantly shaking at $30^{\circ} \mathrm{C}$. Growth curves were fitted in order to extrapolate growth rates that correspond to the maximum slope of the linear range of the $\mathrm{LN}(\mathrm{OD} 600)$ curve over time.

Equipment and settings. Imaging was performed by using a Confocal TCS SP8 and a Confocal TCS SP5 (Leica) equipped with PMT detectors both for fluorescence and transmitted light images. AOBS beam-splitter systems are in place on both instruments. $63 \mathrm{X}$ oil immersion objectives and the LAS AF software were used for all imaging. YFP fluorescence was excited with a $488 \mathrm{~nm}$ laser, while mCherry fluorescence with a $561 \mathrm{~nm}$ laser. Ranges for emission detection were $495-554$ and $637-670 \mathrm{~nm}$ respectively. Image depth is 8-bit in all cases and pixel size equals $120.4 \mathrm{~nm}$. The LUT is linear and covers the full range of the data.

Fluorescence microscopy and image analysis. Yeast cells expressing TDP-43 selected variants were grown in SC-URA $2 \%$ raffinose non-inducing medium and then transferred to SC-URA $2 \%$ galactose medium to induce protein expression for $8 \mathrm{~h}$. They were then imaged under a Confocal TCS SP8 microscope (Leica). Counting of foci was conducted both manually and by automated pipelines using the CellProfiler software where quantification of fluorescent intensity was tracked for each focus. The coordinates of the center of each focus and nucleus were derived from CellProfiler and used to calculate distances using a custom R script (pipelines available at https://github.com/lehner-lab/tardbpdms_cellprofiler_scripts).

Fluorescence recovery after photobleaching. Yeast cells expressing TDP- 43 selected variants were grown in SC-URA $2 \%$ raffinose non-inducing medium and then transferred to SC-URA $2 \%$ galactose medium to induce protein expression for $8 \mathrm{~h}$. The cells were immobilized to an 8-well cover slide by Concanavalin-Amediated cell adhesion. Cells were then imaged under a Confocal TCS SP5 microscope (Leica) where bleaching was achieved with 488 Laser Power at $70 \%$ for three frames (1.3 s per frame) while fluorescence recovery was recorded for 50 frames. The curves were then fitted to a single exponential, following normalization, with the EasyFrap package ${ }^{74}$.

Protein extraction and western blotting. Single yeast colonies were grown overnight in non-inducing medium and then diluted to 0.2 OD600 in galactose medium to induce protein expression for $\sim 8 \mathrm{~h}$. At this stage, $6 \times 10^{7}$ cells were collected and re-suspended in $200 \mu \mathrm{L} \mathrm{EtOH}$ and $2.5 \mu \mathrm{L}$ PMSF. Samples were vortexed with glass beads for $15 \mathrm{~min}$ at $4{ }^{\circ} \mathrm{C}$ and frozen overnight at $-80^{\circ} \mathrm{C}$. The samples were dried in a speed vacuum for $20 \mathrm{~min}$ and resuspended in $200 \mu \mathrm{L}$ solubilizing buffer ( $20 \mathrm{mM}$ Tris $\mathrm{HCl} \mathrm{pH} \mathrm{6.8,2 \%} \mathrm{SDS).} \mathrm{After} \mathrm{boiling} \mathrm{for} 5 \mathrm{~min}$, the lysate fraction was run on a NuPAGE 4-12\% Bis-Tris gels (Novex) and transferred to PVDF membranes in an iBlot (Invitrogen). Membranes were blocked with 5\% milk powder in TBS-T and incubated overnight at $4{ }^{\circ} \mathrm{C}$ with primary antibodies: anti-GFP mouse antibody (Santa Cruz sc-9996) and anti-PGKD1 mouse antibody (Novex 459250) diluted 1:1000 and 1:5000 in $2.5 \%$ powder milk respectively. Secondary antibody anti-proteinG was incubated for $1 \mathrm{~h}$ at room temperature. Proteins were detected with an enhanced chemi-luminescence system (Millipore Luminata) and visualized using an Amersham Imager 600 (GE Healthcare).

Sequencing data pre-processing. FastQ files from paired-end sequencing of replicate deep mutational scanning (DMS) libraries before ('input') and after selection ('output') were processed using a custom pipeline (https://github.com/ lehner-lab/DiMSum, manuscript in prep.). DiMSum is an R package that wraps common biological sequence processing tools including FastQC (http://www. bioinformatics.babraham.ac.uk/projects/fastqc/) (for quality assessment), cutadapt (for demultiplexing and constant region trimming), USEARCH ${ }^{75}$ (for paired-end read alignment) and the FASTX-Toolkit (http://hannonlab.cshl.ed/fastx_toolkit/). First, $5^{\prime}$ constant regions were trimmed, but read pairs were discarded if $5^{\prime}$ constant regions contained more than $20 \%$ mismatches to the reference sequence. Read pairs were aligned (reads that did not match the expected 126 bp length were discarded) and Phred base quality scores of aligned positions were calculated using USEARCH. Reads that contained base calls with Phred scores below 30 (290-331 DMS library) or below 25 (332-373 DMS library) were discarded. Approximately five and seven million reads passed these filtering criteria in each sample corresponding to the 290-331 and 332-373 libraries respectively. Finally, unique variants were counted and merged into a single table of variant counts (aggregated across technical output replicates) per DMS library. One out of four input replicates (and all associated output samples) from each DMS library were discarded due to considerably lower correlations with the other replicates (Supplementary Fig. 1a, b). 
Variant toxicity and error estimates. All analyses of toxicity were performed on variants with a maximum of two AA mutations, but no synonymous mutations in other codons. Firstly, sample-wise counts for variants identical at the AA level were aggregated. For each replicate selection, relative toxicity of variants was calculated from variant counts in input $\left(F_{x_{\text {input }}}\right)$ and output $\left(F_{x_{\text {output }}}\right)$ samples as

$$
\text { Relative toxicity } x_{x}=\mathrm{ES}_{\mathrm{WT}}-\mathrm{ES}_{x}
$$

where $\mathrm{ES}_{x}=\ln \frac{F_{x_{\text {ouput }}}}{F_{x_{\text {xinput }}}}$ and $\mathrm{ES}_{\mathrm{WT}}$ represents the WT enrichment score. Uncertainty of toxicity values was estimated as a combination of expected Poisson error based on read counts and error between replicate selections as:

$$
\varepsilon_{x}=\sqrt{\frac{1}{F_{x_{\text {input }}}}+\frac{1}{F_{x_{\text {output }}}}+\frac{1}{F_{\mathrm{WT}_{\text {input }}}}+\frac{1}{F_{\mathrm{WT}_{\text {output }}}}+\varepsilon_{\mathrm{r}}^{2}} .
$$

Here, $\varepsilon_{r}$, the error between replicate selections, is estimated from the variance of toxicity estimates across replicates for variants whose expected count-based Poisson error approaches zero. Toxicity estimates and associated errors per replicate selection were also normalized by the replicate-specific number of cell doublings during selection to yield relative growth rates per generation.

In 'doped' variant libraries, individual double mutants are represented less frequently than single mutants or the WT sequence and due to this underrepresentation toxic double mutants (that are depleted due to slower growth during selection) are often not observed in the output samples (Supplementary Fig. 1c). To calculate toxicity estimates for such double mutants and avoid skewed marginal toxicity distributions due to these drop-out events, we used a Bayesian approach to estimate toxicity of double mutants based on a prior, i.e., toxicity distributions of highly represented doubles that originate from single mutants with similar toxicity estimates ${ }^{51}$. These corrected toxicity estimates show improved heteroscedasticity and reduced variance, especially for under-represented double mutants

(Supplementary Fig. 1c)

Variant toxicity distributions were first normalized between replicate selections of the same DMS library to have equal standard deviations. Then toxicity estimates of each variant across replicate selections were merged by taking the error-weighted mean across replicate selections. Finally, distributions of merged toxicity estimates from each DMS library were centred on the error-weighted means of toxicity of single codon synonymous (silent) variants in each DMS library and scaled such that the error-weighted means of single STOP codon variants coincided for both DMS libraries (Supplementary Fig. 2a and c). Furthermore, we removed low confidence variants supported by an average of less than ten input reads from all downstream analyses. Merged and normalized toxicity estimates, as well as toxicity estimates from independent replicates before merging and normalisation, are available in Supplementary Data 3 and 4 respectively.

The impact of misreads (i.e. sequencing errors) was evaluated by measuring the per base error frequency in the WT sequence $10 \mathrm{bp}$ upstream and $10 \mathrm{bp}$ downstream of the mutagenized (doped) region. The frequency of an incorrect base call in these regions is $0.0001\left(\mathrm{sd}=6 \times 10^{-5}\right)$ for the 290-331 library and $0.0004\left(\mathrm{sd}=4 \times 10^{-4}\right)$ with little variability depending on the wild-type base. By multiplying these frequencies by the length of the doped region we calculated the probability of a misread in each variant $(0.0126$ for the 290-331 library and 0.0504 for the 332-373 library). Single nucleotide substitutions account for $\sim 2 \times$ $10^{6}$ reads in a typical input sample of the 290-331 library, of which we estimate $98.74 \%$ to be "true" single nucleotide variants on the basis of a 0.0126 misread probability. Therefore, we estimate an additional $2 \times 10^{4}$ misreads originate from single nucleotide variants $\left(2 \times 10^{4}=0.0126 / 0.9874 \times 2 \times 10^{6}\right)$. In the $126 \mathrm{bp}$ mutagenized region, a total of $7875 \times 3 \times 3=\sim 7 \times 10^{4}$ possible double nucleotide variants exist, since each base in each pair can be mutated to one of the three other nucleotides. We therefore estimated that, even in a scenario in which single nucleotide variants are solely distributed among all possible double nucleotide variants, the additional count due to sequencing errors in the $290-331$ library would be $\sim 0.5$ as it follows from the estimated additional $2.6 \times$ $10^{4}$ misreads over a total of $7 \times 10^{4}$ possible doubles. Similarly, additional counts due to sequencing errors would not reach 2 even in the 332-373 library, where the misread frequency was higher $(0.0004)$.

Linear regression models to predict variant toxicity. We used simple linear regression to predict variant toxicity from (i) a collection of AA property features, (ii) a panel of scores from aggregation/structure algorithms and (iii) location with respect to the toxicity hotspot.

The AA property features were derived from a PCA of a curated collection of numerical indices representing various physicochemical and biochemical properties of AAs (http://www.genome.jp/aaindex/). From a total of 539 indices, we retained 379 high confidence indices with no missing values (including five additional indices absent from the original database; see Supplementary Data 2). Results of PCA and selected variable loadings on the normalized matrix are shown in Supplementary Fig. 3. For single mutant variants, AA property feature values represent the difference between the WT and mutant PC scores.

Similarly, aggregation, disorder, structure and other feature values for single mutant variants represent the difference between scores obtained using WT and single mutant AA sequences. AGADIR, catGRANULE and Tango provide a single score per AA sequence. Unless a single score per AA sequence was provided (i.e. AGADIR, catGRANULE, Tango), individual residue-level scores were summed to obtain a score per AA sequence (i.e. BetaTPred3, DISEMBL, IUPred2A, Waltz, ZipperDB, Zyggregator). The entire PRD AA sequence was supplied to AGADIR and all unique six-mers to ZipperDB. For the remainder, the full-length AA sequence was used.

Variants inside the hotspot were defined as those with mutant residue positions in the range of 312-342. Change in absolute charge (regardless of sign) is shown in Fig. $2 \mathrm{~d}$, e, because this feature is more predictive of toxicity than change in charge itself. For double mutant variants, we summed the feature values of the constituent singles for both AA property and aggregation/structure algorithm features. Regression models were built using either (i) all variants, restricting variants to those occurring either (ii) inside or (iii) outside the toxicity hotspot (for double mutants both mutations have to occur either inside or outside the hotspot region), or (iv) including a binary location variable (0: one/all outside, 1: one inside, one outside, 2: one/all inside toxicity hotspot) and a third term indicating the interaction between location and the AA property or aggregation/structure algorithm feature.

Predicting secondary structure from epistasis. Epistasis is the nonindependence of mutation effects, i.e., the toxicity of double mutants is different from that expected given the toxicity of their constituent single mutant variants. We have previously shown that epistasis between double mutants can result from structural interactions within proteins and therefore can be used to infer secondary and tertiary structural features ${ }^{51,60}$. In brief, double mutants were classified as epistatic if they had more extreme toxicity values (below 5th percentile or above 95th percentile) than other double mutants with similar single mutant toxicities, which was estimated from non-parametric surface fits of double mutant toxicity as a function of a two-dimensional single mutant toxicity space (Fig. 4a).

Double mutants close to the lower or upper measurement range limits (where the power to detect significant epistasis is reduced) were excluded from epistasis quantification. We calculated position-pair enrichments for epistatic double mutants resulting in a pair-wise enrichment matrix. Diagonal entries on this matrix were imputed as column-wise mean enrichments. An epistasis correlation score matrix was then derived from this enrichment matrix by calculating the partial correlation of epistasis interaction profiles (columns of the enrichment matrix) between all pairs of positions. The rationale for the correlation score is that structurally close positions within a protein should have similar epistatic interactions with all other positions in the protein. Calculating partial correlations additionally removes transitive interactions and was found to be superior over epistasis enrichments in estimating secondary structures ${ }^{51}$.

Secondary structure propensities were calculated by testing for agreement of epistasis correlation score patterns with the stereotypical periodicities of an $\alpha$-helix and $\beta$-strand, using two-dimensional kernels at each position along the diagonal of the epistasis correlation score matrix ${ }^{51}$. Significance of secondary structure propensities was assessed by comparison to propensities derived from $10^{4}$ randomized epistasis correlation score matrices.

Similarly, LARKS structure propensities were calculated using PDB-structure derived contact matrices based on a minimal side-chain heavy atom distance of $4.5 \AA$ (Supplementary Fig. 8) for both WT (PDB 5WHN [https://www.rcsb.org/ structure/5WHN]) and mutant sequences (PDB 5WHP [https://www.rcsb.org/ structure/5WHP] and 5WKB [https://www.rcsb.org/structure/5WKB]). Contact matrix values were normalised to have zero sum. Association score matrix values were normalised to have mean of zero and unit variance. Significance of LARKS structure propensities was assessed by comparison to propensities derived from $10^{4}$ randomized epistasis correlation score matrices, where randomization was restricted to within-LARKS interactions, i.e., distances compatible with a six-mer

Reporting summary. Further information on research design is available in the Nature Research Reporting Summary linked to this article.

\section{Data availability}

The data that support the findings of this study are available from the corresponding author upon request. Raw sequencing data and the processed data table (Supplementary Data 3) have been deposited in NCBI's Gene Expression Omnibus (GEO) and are accessible through the GEO Series accession number GSE128165. The source data underlying Fig. 3 and Supplementary Figs. 5 and 6 are provided as a Source Data file.

\section{Code availability}

All software code and custom scripts are available on GitHub: https:/github.com/lehnerlab/DiMSum for raw read processing, https://github.com/lehner-lab/tardbpdms for all downstream analyses and to produce all figures, and https://github.com/lehner-lab/ tardbpdms_cellprofiler_scripts for CellProfiler pipelines.

Received: 20 May 2019 Accepted: 15 August 2019 Published online: 13 September 2019 


\section{References}

1. Buratti, E. Functional Significance of TDP-43 Mutations in Disease. Adv Genet. 91, 1-53 (2015)

2. $\quad$ Ling, S.-C., Polymenidou, M. \& Cleveland, D. W. Converging mechanisms in ALS and FTD: disrupted RNA and protein homeostasis. Neuron 79, 416-438 (2013).

3. Chiti, F., Stefani, M., Taddei, N., Ramponi, G. \& Dobson, C. M. Rationalization of the effects of mutations on peptide and protein aggregation rates. Nature 424, 805-808 (2003).

4. Eisenberg, D. \& Jucker, M. The amyloid state of proteins in human diseases. Cell 148, 1188-1203 (2012).

5. Chiti, F. \& Dobson, C. M. Protein misfolding, functional amyloid, and human disease. Annu. Rev. Biochem. 75, 333-366 (2006)

6. Polymeropoulos, M. H. et al. Mutation in the alpha-synuclein gene identified in families with Parkinson's disease. Science 276, 2045-2047 (1997).

7. Arnold, E. S. et al. ALS-linked TDP-43 mutations produce aberrant RNA splicing and adult-onset motor neuron disease without aggregation or loss of nuclear TDP-43. Proc. Natl Acad. Sci. USA 110, E736-E745 (2013)

8. Taylor, J. P., Brown, R. H. \& Cleveland, D. W. Decoding ALS: from genes to mechanism. Nature 539, 197-206 (2016)

9. Gordon, D. et al. Single-copy expression of an amyotrophic lateral sclerosislinked TDP-43 mutation (M337V) in BAC transgenic mice leads to altered stress granule dynamics and progressive motor dysfunction. Neurobiol. Dis. 121, 148-162 (2019).

10. De Strooper, B. Lessons from a failed $\gamma$-secretase Alzheimer trial. Cell 159, 721-726 (2014).

11. Karran, E., Mercken, M. \& Strooper, B. D. The amyloid cascade hypothesis for Alzheimer's disease: an appraisal for the development of therapeutics. Nat. Rev. Drug Discov. 10, 698 (2011)

12. Mitsumoto, H., Brooks, B. R. \& Silani, V. Clinical trials in amyotrophic lateral sclerosis: why so many negative trials and how can trials be improved? Lancet Neurol. 13, 1127-1138 (2014).

13. Bolognesi, B. et al. ANS binding reveals common features of cytotoxic amyloid species. ACS Chem. Biol. 5, 735-740 (2010).

14. Cremades, N. et al. Direct observation of the interconversion of normal and toxic forms of a-synuclein. Cell 149, 1048-1059 (2012).

15. Fang, Y.-S. et al. Full-length TDP-43 forms toxic amyloid oligomers that are present in frontotemporal lobar dementia-TDP patients. Nat. Commun. 5, 4824 (2014).

16. Zhu, C., Beck, M. V., Griffith, J. D., Deshmukh, M. \& Dokholyan, N. V. Large SOD1 aggregates, unlike trimeric SOD1, do not impact cell viability in a model of amyotrophic lateral sclerosis. Proc. Natl Acad. Sci. USA. 115, 4661-4665 (2018).

17. Escusa-Toret, S., Vonk, W. I. M. \& Frydman, J. Spatial sequestration of misfolded proteins by a dynamic chaperone pathway enhances cellular fitness during stress. Nat. Cell Biol. 15, 1231-1243 (2013).

18. Mateju, D. et al. An aberrant phase transition of stress granules triggered by misfolded protein and prevented by chaperone function. EMBO J. 36, 1669-1687 (2017).

19. Alberti, S. \& Hyman, A. A. Are aberrant phase transitions a driver of cellular aging? Bioessays 38, 959-968 (2016).

20. Khan, T. et al. Quantifying nucleation in vivo reveals the physical basis of prion-like phase behavior. Mol. Cell 71, 155-168.e7 (2018).

21. Guo, L. et al. Nuclear-import receptors reverse aberrant phase transitions of RNA-binding proteins with prion-like domains. Cell 173, 677-692.e20 (2018).

22. Franzmann, T. M. et al. Phase separation of a yeast prion protein promotes cellular fitness. Science 359, eaao5654 (2018).

23. Wang, J. et al. A molecular grammar governing the driving forces for phase separation of prion-like RNA binding proteins. Cell 174, 688-699.e16 (2018).

24. Patel, A. et al. A liquid-to-solid phase transition of the ALS protein FUS accelerated by disease mutation. Cell 162, 1066-1077 (2015).

25. Molliex, A. et al. Phase separation by low complexity domains promotes stress granule assembly and drives pathological fibrillization. Cell 163, 123-133 (2015).

26. Wegmann, S. et al. Tau protein liquid-liquid phase separation can initiate tau aggregation. EMBO J. 37, e98049 (2018).

27. Vavouri, T., Semple, J. I., Garcia-Verdugo, R. \& Lehner, B. Intrinsic protein disorder and interaction promiscuity are widely associated with dosage sensitivity. Cell 138, 198-208 (2009).

28. Bolognesi, B. et al. A concentration-dependent liquid phase separation can cause toxicity upon increased protein expression. Cell Rep. 16, 222-231 (2016).

29. Halfmann, R. et al. Opposing effects of glutamine and asparagine govern prion formation by intrinsically disordered proteins. Mol. Cell 43, 72-84 (2011).

30. Neumann, M. et al. Ubiquitinated TDP-43 in frontotemporal lobar degeneration and amyotrophic lateral sclerosis. Science 314, 130-133 (2006).
31. Higashi, S. et al. Concurrence of TDP-43, tau and alpha-synuclein pathology in brains of Alzheimer's disease and dementia with Lewy bodies. Brain Res. 1184, 284-294 (2007).

32. Schwab, C., Arai, T., Hasegawa, M., Yu, S. \& McGeer, P. L. Colocalization of transactivation-responsive DNA-binding protein 43 and Huntingtin in inclusions of Huntington disease. J. Neuropathol. Exp. Neurol. 67, 1159-1165 (2008).

33. Amador-Ortiz, C. et al. TDP-43 immunoreactivity in hippocampal sclerosis and Alzheimer's disease. Ann. Neurol. 61, 435-445 (2007).

34. Uchino, A. et al. Incidence and extent of TDP-43 accumulation in aging human brain. Acta Neuropathol. Commun. 3, 35 (2015).

35. Jiang, L.-L. et al. Structural transformation of the amyloidogenic core region of TDP-43 protein initiates its aggregation and cytoplasmic inclusion. J. Biol. Chem. 288, 19614-19624 (2013).

36. Conicella, A. E., Zerze, G. H., Mittal, J. \& Fawzi, N. L. ALS mutations disrupt phase separation mediated by $\alpha$-helical structure in the TDP-43 lowcomplexity C-terminal domain. Struct./Fold. Des. 24, 1537-1549 (2016).

37. Sun, Y. \& Chakrabartty, A. Phase to phase with TDP-43. Biochemistry 56, 809-823 (2017)

38. Schmidt, H. B., Barreau, A. \& Rohatgi, R. Decoding and recoding phase behavior of TDP43 reveals that phase separation is not required for splicing function. Preprint at https://www.biorxiv.org/content/10.1101/548339v1 (2019).

39. Babinchak, W. M. et al. The role of liquid-liquid phase separation in aggregation of the TDP-43 low complexity domain. J. Biol. Chem. (2019). https://doi.org/10.1074/jbc.RA118.007222

40. Sreedharan, J. et al. TDP-43 mutations in familial and sporadic amyotrophic lateral sclerosis. Science 319, 1668-1672 (2008).

41. Chou, C.-C. et al. TDP-43 pathology disrupts nuclear pore complexes and nucleocytoplasmic transport in ALS/FTD. Nat. Neurosci. 21, 228-239 (2018).

42. McGurk, L. et al. Poly(ADP-Ribose) Prevents pathological phase separation of TDP-43 by promoting liquid demixing and stress granule localization. Mol. Cell 71, 703-717 (2018)

43. Coyne, A. N. et al. Fragile X protein mitigates TDP-43 toxicity by remodeling RNA granules and restoring translation. Hum. Mol. Genet. 24, 6886-6898 (2015).

44. Gasset-Rosa, F. et al. Cytoplasmic TDP-43 de-mixing independent of stress granules drives inhibition of nuclear import, loss of nuclear TDP-43, and cell death. Neuron 102, 339-357 (2019).

45. D'Alton, S. et al. Divergent phenotypes in mutant TDP-43 transgenic mice highlight potential confounds in TDP-43 transgenic modeling. PLoS ONE 9, e86513 (2014)

46. Mann, J. R. et al. RNA binding antagonizes neurotoxic phase transitions of TDP-43. Neuron 102, 321-338 (2019).

47. Jiang, L.-L. et al. Two mutations G335D and Q343R within the amyloidogenic core region of TDP-43 influence its aggregation and inclusion formation. Sci. Rep. 6, 23928 (2016).

48. Fowler, D. M. \& Fields, S. Deep mutational scanning: a new style of protein science. Nat. Methods 11, 801 (2014).

49. Staller, M. V. et al. A high-throughput mutational scan of an intrinsically disordered acidic transcriptional activation domain. Cell Syst. 6, 444-455.e6 (2018).

50. Ravarani, C. N. et al. High-throughput discovery of functional disordered regions: investigation of transactivation domains. Mol. Syst. Biol. 14, e8190 (2018).

51. Schmiedel, J. M. \& Lehner, B. Determining protein structure using deep mutagenesis. Nat. Genet. 51, 1177-1186 (2019).

52. Guenther, E. L. et al. Atomic structures of TDP-43 LCD segments and insights into reversible or pathogenic aggregation. Nat. Struct. Mol. Biol. 25, 463-471 (2018).

53. Mompeán, M. et al. Structural evidence of amyloid fibril formation in the putative aggregation domain of TDP-43. J. Phys. Chem. Lett. 6, 2608-2615 (2015).

54. Johnson, B. S. et al. TDP-43 is intrinsically aggregation-prone, and amyotrophic lateral sclerosis-linked mutations accelerate aggregation and increase toxicity. J. Biol. Chem. 284, 20329-20339 (2009).

55. Johnson, B. S., McCaffery, J. M., Lindquist, S. \& Gitler, A. D. A yeast TDP-43 proteinopathy model: exploring the molecular determinants of TDP-43 aggregation and cellular toxicity. Proc. Natl Acad. Sci. USA 105, 6439-6444 (2008).

56. Tamaki, Y. et al. Elimination of TDP-43 inclusions linked to amyotrophic lateral sclerosis by a misfolding-specific intrabody with dual proteolytic signals. Sci. Rep. 8, 6030 (2018)

57. Farrawell, N. E. et al. Distinct partitioning of ALS associated TDP-43, FUS and SOD1 mutants into cellular inclusions. Sci. Rep. 5, 13416 (2015).

58. Kaganovich, D., Kopito, R. \& Frydman, J. Misfolded proteins partition between two distinct quality control compartments. Nature 454, 1088-1095 (2008). 
59. Peskett, T. R. et al. A liquid to solid phase transition underlying pathological Huntingtin Exon1 aggregation. Mol. Cell 70, 588-601.e6 (2018).

60. Rollins, N. J. et al. Inferring protein 3D structure from deep mutation scans. Nat. Genet. 51, 1170-1176 (2019).

61. Toth-Petroczy, A. et al. Structured states of disordered proteins from genomic sequences. Cell 167, 158-170.e12 (2016).

62. Hughes, M. P. et al. Atomic structures of low-complexity protein segments reveal kinked $\beta$ sheets that assemble networks. Science 359, 698-701 (2018).

63. Lim, L., Wei, Y., Lu, Y. \& Song, J. ALS-causing mutations significantly perturb the self-assembly and interaction with nucleic acid of the intrinsically disordered prion-like domain of TDP-43. PLoS Biol. 14, e1002338 (2016).

64. Chiti, F. \& Dobson, C. M. Protein misfolding, amyloid formation, and human disease: a summary of progress over the last decade. Annu. Rev. Biochem. 86, 27-68 (2017)

65. Armakola, M. et al. Inhibition of RNA lariat debranching enzyme suppresses TDP-43 toxicity in ALS disease models. Nat. Genet. 44, 1302-1309 (2012).

66. Weisberg, S. J. et al. Compartmentalization of superoxide dismutase 1 (SOD1G93A) aggregates determines their toxicity. Proc. Natl Acad. Sci. USA 109, 15811-15816 (2012)

67. Arrasate, M., Mitra, S., Schweitzer, E. S., Segal, M. R. \& Finkbeiner, S. Inclusion body formation reduces levels of mutant huntingtin and the risk of neuronal death. Nature 431, 805-810 (2004).

68. Collinge, J. Mammalian prions and their wider relevance in neurodegenerative diseases. Nature 539, 217-226 (2016).

69. Knowles, T. P. J., Vendruscolo, M. \& Dobson, C. M. The amyloid state and its association with protein misfolding diseases. Nat. Rev. Mol. Cell Biol. 15, 384-396 (2014).

70. Karran, E. \& De Strooper, B. The amyloid cascade hypothesis: are we poised for success or failure? J. Neurochem. 139, 237-252 (2016).

71. Chiò, A. et al. Lithium carbonate in amyotrophic lateral sclerosis: lack of efficacy in a dose-finding trial. Neurology 75, 619-625 (2010).

72. Dupuis, L. et al. A randomized, double blind, placebo-controlled trial of pioglitazone in combination with riluzole in amyotrophic lateral sclerosis. PLOS ONE 7, e37885 (2012).

73. Kitzman, J. O., Starita, L. M., Lo, R. S., Fields, S. \& Shendure, J. Massively parallel single-amino-acid mutagenesis. Nat. Methods 12, 203-206 (2015).

74. Rapsomaniki, M. A. et al. easyFRAP: an interactive, easy-to-use tool for qualitative and quantitative analysis of FRAP data. Bioinformatics $\mathbf{2 8}$ 1800-1801 (2012).

75. Edgar, R. C. Search and clustering orders of magnitude faster than BLAST. Bioinformatics 26, 2460-2461 (2010).

\section{Acknowledgements}

Work in B.L.'s lab was supported by a European Research Council (ERC) Consolidator grant (616434), the Spanish Ministry of Economy and Competitiveness (BFU2017-

89488-P), the AXA Research Fund, the Bettencourt Schueller Foundation, and Agencia de Gestio d'Ajuts Universitaris i de Recerca (AGAUR, SGR-831) G.G.T.'s lab was supported by the European Research Council (RIBOMYLOME_309545) and the Spanish Ministry of Economy and Competitiveness (BFU2014-55054-P and BFU2017-86970-P) We acknowledge support from the Spanish Ministry of Economy and Competitiveness, 'Centro de Excelencia Severo Ochoa 2013-2017', the EMBL Partnership, and the CERCA Program/Generalitat de Catalunya. We thank Pablo Baeza Centurión, Xavier Salvatella, Alexandros Armaos and Benjamin Lang for discussion and assistance and the Eisenberg lab for help with the ZipperDB analysis.

\section{Author contributions}

B.B. and B.L. conceived the project and designed the experiments; B.B. and M.S. performed the experiments; A.J.F., B.B. and J.M.S. performed analyses of sequences; A.J.F. and J.M.S. analysed the genetic interactions and structures; G.G.T. initiated, designed and carried out the original computational analysis of physicochemical properties; B.B., A.J.F. and B.L. wrote the manuscript with input from all authors.

\section{Additional information}

Supplementary Information accompanies this paper at https://doi.org/10.1038/s41467019-12101-z.

Competing interests: The authors declare no competing interests.

Reprints and permission information is available online at http://npg.nature.com/ reprintsandpermissions/

Peer review information Nature Communications thanks the anonymous reviewers for their contribution to the peer review of this work. Peer reviewer reports are available.

Publisher's note Springer Nature remains neutral with regard to jurisdictional claims in published maps and institutional affiliations.

Open Access This article is licensed under a Creative Commons Attribution 4.0 International License, which permits use, sharing, adaptation, distribution and reproduction in any medium or format, as long as you give appropriate credit to the original author(s) and the source, provide a link to the Creative Commons license, and indicate if changes were made. The images or other third party material in this article are included in the article's Creative Commons license, unless indicated otherwise in a credit line to the material. If material is not included in the article's Creative Commons license and your intended use is not permitted by statutory regulation or exceeds the permitted use, you will need to obtain permission directly from the copyright holder. To view a copy of this license, visit http://creativecommons.org/ licenses/by/4.0/

(C) The Author(s) 2019 